\title{
Frequency-domain analysis of intrinsic neuronal properties using high-resistant electrodes
}

\author{
Christian Rössert ${ }^{1,2 *}$, Hans Straka ${ }^{3}$, Stefan Glasauer ${ }^{1,4}$ and Lee E. Moore ${ }^{3}$ \\ Institute for Clinical Neurosciences, Ludwig-Maximilians-Universität München, Munich, Germany \\ 2 Graduate School of Systemic Neurosciences, Ludwig-Maximilians-Universität München, Munich, Germany \\ ${ }^{3}$ Laboratoire de Neurobiologie des Réseaux Sensorimoteurs, Centre National de la Recherche Scientifique, UMR 7060, Université Paris Descartes, Paris, France \\ ${ }^{4}$ Bernstein Center for Computational Neuroscience Munich, Munich, Germany
}

Edited by:

Laszlo Acsady, Institute of

Experimental Medicine, Hungary

\section{Reviewed by:}

Victor Varga, Institute of Experimental

Medicine of the Hungarian Academy of

Sciences, Hungary

Marco Diana, Ecole Normale

Supérieure, France

Robert Renden, Heidelberg University,

Germany

${ }^{*}$ Correspondence:

Christian Rössert, Klinikum der

Universität München, Zentrum für

Sensomotorik, Marchioninistr. 23,

81377 München, Germany.

e-mail: roessert@Irz.uni-muenchen.de
Intrinsic cellular properties of neurons in culture or slices are usually studied by the whole cell clamp method using low-resistant patch pipettes. These electrodes allow detailed analyses with standard electrophysiological methods such as current- or voltage-clamp. However, in these preparations large parts of the network and dendritic structures may be removed, thus preventing an adequate study of synaptic signal processing. Therefore, intact in vivo preparations or isolated in vitro whole brains have been used in which intracellular recordings are usually made with sharp, high-resistant electrodes to optimize the impalement of neurons. The general nonlinear resistance properties of these electrodes, however, severely limit accurate quantitative studies of membrane dynamics especially needed for precise modelling. Therefore, we have developed a frequency-domain analysis of membrane properties that uses a Piece-wise Nonlinear Electrode Compensation (PNEC) method. The technique was tested in second-order vestibular neurons and abducens motoneurons of isolated frog whole brain preparations using sharp potassium chloride- or potassium acetate-filled electrodes. All recordings were performed without online electrode compensation. The properties of each electrode were determined separately after the neuronal recordings and were used in the frequency-domain analysis of the combined measurement of electrode and cell. This allowed detailed analysis of membrane properties in the frequency-domain with high-resistant electrodes and provided quantitative data that can be further used to model channel kinetics. Thus, sharp electrodes can be used for the characterization of intrinsic properties and synaptic inputs of neurons in intact brains.

Keywords: vestibular, abducens, model, intracellular, capacitance, resistance, ion-channel, electrode compensation

\section{INTRODUCTION}

The standard method for characterizing properties of single neurons is the intracellular recording with glass micropipettes. Quantitative data on intrinsic and synaptic properties have been mainly obtained from recordings of neurons in culture or in slice preparations with low-resistant patch pipettes. The use of these low-resistant electrodes is required for the voltage clamp control of the membrane potential, which has been used for detailed analyses of ion channel kinetics (Neher and Sakmann, 1976). However, a major drawback of these reduced preparations is that the surrounding neuronal network and the dendritic structures of individual neurons are partially removed, thus making this experimental approach less appropriate for an investigation of synaptic signal processing in complex circuits with feed-forward and feed-back loops, in particular where multiple and longerrange circuits contribute to this processing. Therefore, spatial and temporal aspects of synaptic signal processing in single neurons have been frequently studied in vivo or in isolated in vitro vertebrate whole brain preparations (e.g. Llinás and Yarom, 1981; Hounsgaard et al., 1988; Babalian et al., 1997). In these preparations, intracellular recordings of neurons are most efficiently made with high-resistant $(\sim 50-120 \mathrm{M} \Omega)$ sharp glass electrodes to maximize the success rate of impaling neurons.
The disadvantage of these high-resistant sharp electrodes, however, is that it is necessary to compensate the non-linear voltage drop across the electrode during intracellular current injections. The electrode compensation circuits that are implemented in most intracellular amplifiers usually treat the electrode as a simple linear $\mathrm{RC}$ circuit (resistor and capacitor). This procedure, however, is generally inadequate since sharp electrodes are often not simple $\mathrm{RC}$ elements and show current-dependent non-linear resistance changes (Brette et al., 2008) that are difficult to describe quantitatively and thus impair a reliable use of bridge compensation (BC) or discontinuous current clamp (DCC) compensation (Moore et al., 1993).

The present study describes a novel frequency-domain analysis of single neurons using offline electrode compensation that employs a Piece-wise Non-linear Electrode Compensation (PNEC) procedure to remove the separately measured electrode from the combination of both electrode and cell impedance. With this method it is possible to compensate for arbitrarily complex electrodes in frequency-domain data, which is an improvement to BC and DCC. Moreover it is also an improvement to the novel Active Electrode Compensation (AEC) method (Brette et al., 2008) since the PNEC does not rely on the resistance linearity of the electrode and is independent of the ratio of electrode and membrane time constants, 
which do not need to be estimated mathematically from combined measurements of electrode and neuron.

The frequency-domain data provide current-dependent transfer functions, which can be used for the characterization of intrinsic membrane properties or to directly fit compartmental models with a comparable precision and reliability as those obtained from patchclamp measurements (Booth et al., 1997; Tennigkeit et al., 1998; Roth and Häusser, 2001; Erchova et al., 2004; Taylor and Enoka, 2004; Idoux et al., 2008). Preliminary results have been published in abstract form (Rössert et al., 2008).

\section{MATERIALS AND METHODS WHOLE BRAIN PREPARATION}

In vitro experiments were performed on isolated brains of six adult grass frogs (Rana temporaria) and complied with the "Principles of animal care", publication No. 86-23, revised 1985 by the National Institute of Health. As described in previous studies (Straka and Dieringer, 1993), animals were deeply anesthetized with $0.1 \%$ 3-aminobenzoic acid ethyl ester (MS-222), and perfused transcardially with iced Ringer solution ( $75 \mathrm{mM} \mathrm{NaCl} ; 25 \mathrm{mM} \mathrm{NaHCO}_{3}$; $2 \mathrm{mM} \mathrm{CaCl}_{2} ; 2 \mathrm{mM} \mathrm{KCl} ; 0.5 \mathrm{mM} \mathrm{MgCl}_{2} ; 11 \mathrm{mM}$ glucose; $\mathrm{pH} 7.4$ ). Thereafter, the skull and bony labyrinth were opened ventrally. After dissecting the three semicircular canals on each side, the brain was removed with all labyrinthine end organs attached to the VIIIth nerve. Subsequently, the brain was submerged in iced Ringer and the dura, the labyrinthine end organs and the choroid plexus covering the IVth ventricle were removed. In all experiments the forebrain was disconnected. Brains were used as long as 4 days after their isolation and were stored overnight at $6^{\circ} \mathrm{C}$ in continuously oxygenated Ringer solution (Carbogen: $95 \% \mathrm{O}_{2}, 5 \% \mathrm{CO}_{2}$ ) with a $\mathrm{pH}$ of $7.4 \pm 0.1$. The brains were directly fixed with insect pins to the sylgard floor of a chamber (volume $2.4 \mathrm{ml}$ ) or glued with cyanoacrylate to a plastic mesh that was fixed to the floor of the recording chamber. The chamber was continuously perfused with oxygenated Ringer solution at a rate of $1.3-2.1 \mathrm{ml} / \mathrm{min}$. The temperature was electronically controlled and maintained at $14 \pm 0.1^{\circ} \mathrm{C}$.

\section{CLASSIFICATION OF NEURONAL CELL TYPES}

External landmarks of the isolated frog whole brain served to identify the target sites for the intracellular recordings in the vestibular (Pfanzelt et al., 2008) and the abducens nuclei (Straka and Dieringer, 1993). Intracellularly recorded second-order vestibular neurons $\left(2^{\circ} \mathrm{VN}\right)$ were identified by the activation of monosynaptic EPSPs following electrical stimulation of individual ipsilateral semicircular canal nerves (Straka et al., 1997) with single constant current pulses (duration: $0.2 \mathrm{~ms}$; threshold: $\sim 1-3 \mu \mathrm{A}$ ). These pulses were produced by a stimulus isolation unit (WPI A 360) and applied across suction electrodes whose opening diameters $(120-150 \mu \mathrm{m})$ were individually adjusted. All $2^{\circ} \mathrm{VN}$ were classified as phasic or tonic neurons based on their responses to the injection of long, positive current steps (Straka et al., 2004; Beraneck et al., 2007; Pfanzelt et al., 2008). Abducens motoneurons (AbMot) were identified by short-latency antidromic action potentials following electrical stimulation of the ipsilateral VIth cranial nerve with suction electrodes (diameter 100-150 $\mu \mathrm{m}$ ). These neurons typically received a disynaptic crossed EPSP and an uncrossed IPSP following electrical stimulation of the bilateral horizontal canal nerve, compatible with previous results obtained after electrical stimulation of the entire VIIIth nerve (Straka and Dieringer, 1993). Only $2^{\circ} \mathrm{VN}$ and $\mathrm{AbMot}$ with resting membrane potentials that were more negative than $-55 \mathrm{mV}$ were included in this study.

\section{COMPLEX ADMITTANCE MEASUREMENTS WITH SHARP MICROELECTRODES}

Sharp high-resistant glass microelectrodes for intracellular recordings were made with a horizontal puller (P-87 Brown/Flaming) using filament-containing borosilicate glass with pre-fire-polished ends (GB150F-8P, Science Products GmbH, Hofheim, Germany). Electrodes were filled with a 3-M solution of $\mathrm{KCl}$ or a mixture of $3 \mathrm{M}$ $\mathrm{KCl}$ and $2 \mathrm{M} \mathrm{KAc} \mathrm{(1:10),} \mathrm{which} \mathrm{gave} \mathrm{final} \mathrm{resistances} \mathrm{of} \mathrm{70-90} \mathrm{M} \Omega$ and $80-120 \mathrm{M} \Omega$, respectively. Bath application of the potassium channel blocker 4-aminopyridine (4-AP; $20 \mu \mathrm{M})$ confirmed in one experiment the putative contribution of voltage-dependent potassium conductances (Wu et al., 2001; Beraneck et al., 2007) to the non-linear response properties of phasic $2^{\circ} \mathrm{VN}$.

Voltage recordings and current injections were performed with a single-electrode clamp amplifier (SEC-05L, NPI Electronic GmbH, Tamm, Germany) in the "bridge balance" operation mode with no resistance or capacitance compensation. For A/D and D/A conversion a National Instruments acquisition card (PCI-6052E) was used with a PC (Intel Pentium 4, 1.9 GHz, Windows XP); the signals were sampled at $5 \mathrm{kHz}$. Recordings were done with a custom Matlab (Mathworks Corp.) program.

Responses of $2^{\circ} \mathrm{VN}$ and AbMot to a multi-sine current-clamp were measured at different membrane potentials. The multi-sine stimulus $I_{\mathrm{MS}}$ was composed of 52-55 discrete frequencies of interest with a uniform stimulus amplitude and randomized phase spectra over a range of either $0.2-988.8 \mathrm{~Hz}$ or $0.2-1935.7 \mathrm{~Hz}$ (Idoux et al., 2008). The constant magnitude was designed to drive the voltage responses uniformly at all frequencies of interest and the random phase spectrum was chosen to minimize the peak-to-peak dynamic amplitude of the stimulus waveform. The maximal half-amplitude of the multi-sine stimulus ( $h_{\mathrm{Amp}}=$ half of peak-to-peak amplitude) ranged from 0.4 to $1.2 \mathrm{nA}$ with a duration of 6 or $12 \mathrm{~s}$. In order to measure neurons at membrane potentials of interest (just below spike threshold), the multi-sine stimulus $I_{\mathrm{MS}}$ was superimposed on a constant holding current $I_{\text {hold }}$, which preceded the multi-sine stimulus by $1.5 \mathrm{~s}$. This holding current + multi-sine signal was the current clamp command or input current, $I_{\text {ref }}(t)$, (Figure 1B) that led to an output voltage response $V(t)$ (Figure 1C). Each measurement consisted of two stimuli, where the second multisine stimulus, $I_{\mathrm{MS}}$, was multiplied by -1 . A fast Fourier transform (FFT) was done on the difference of the two runs providing $I_{\text {ref }}(f)$ and $V(f)$, respectively. The FFT, without windowing, has been computed on all $N$ data points of $I_{\text {ref }}(f)$ and $V(f)$, but only the frequencies present in the stimulus were used for the frequencydomain analysis.

The sum of the voltage responses $V(t), V_{\mathrm{AveM}}$, revealed the mean membrane potential and non-linear membrane effects, such as subthreshold responses or action potentials, as well as recurrent synaptic potentials evoked by the current injection during the two runs. Since the polarity of the stimulus alternated, the sum removed all coherent linear responses and thus $V_{\mathrm{AveM}}$ is an excellent control for the quality of the measurement. The corresponding admittance 


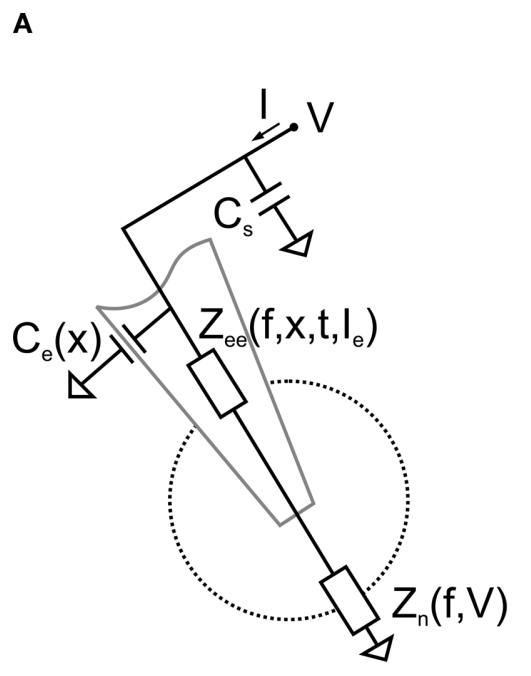

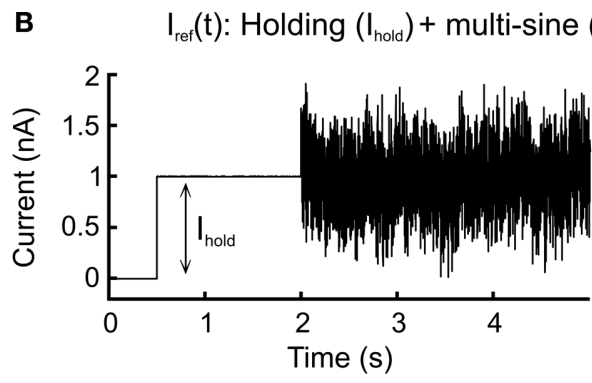

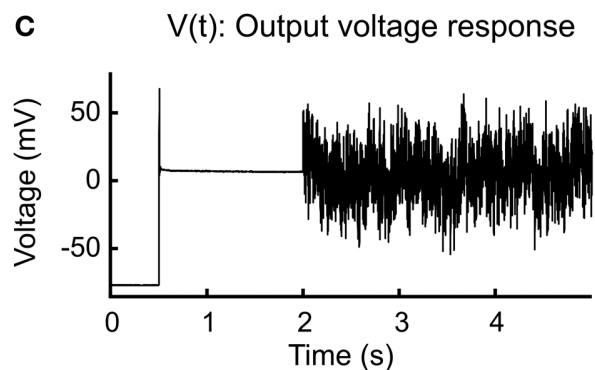

$\mathrm{FFT} \rightarrow \mathrm{I}_{\text {ref }}(\mathrm{f})$

$$
Y(f)=\frac{l_{\text {ref }}(f)}{V(f)}
$$

$\mathrm{FFT} \rightarrow \mathrm{V}(\mathrm{f})$
FIGURE 1 | Circuit diagram and multi-sine measurement procedure. (A) Equivalent circuit diagram of a sharp electrode inside a neuron. The neuron is described as a frequency- and voltage-dependent impedance, $Z_{n}(f, V)$; the electrode is described as a frequency-, depth-, time- and current-dependent impedance $Z_{\mathrm{ee}}\left(f, x, t, l_{\mathrm{e}}\right)$ serial to and a depth-dependent capacitance $C_{\mathrm{e}}(x)$ parallel to the neuron; additional constant parallel stray capacitance $C_{\mathrm{s}}$ of amplifier circuit. (B,C) Multi-sine current stimulus with discrete frequencies superimposed on a constant holding current, $I_{\text {hold }}(\mathbf{B})$, injected through the electrode leading to a voltage response (C). Transformation into the frequency-domain by a fast Fourier transform (FFT) and division of the stimulus current by the voltage response leads to the admittance transfer function, $Y(f)$. transfer functions were computed as $Y(f)=I_{\text {ref }}(f) / V(f)$. Admittance extends the concept of conductance, taking into account dynamic effects by describing not only the relative amplitudes of the voltage and current, but also the relative phases. Admittance is expressed as a complex number $Y(f)=G+j B$ with $j$ being the imaginary unit and $G$ the real part. It is the inverse of the impedance $Z(f)=1 / Y(f)$, which is also a complex number, $Z=R+j C$ with resistance $R$ being the real part.

All transfer functions are shown as complex admittance or impedance Bode plots. In the complex admittance plots, the real part is shown on the $x$-axis, whereas the imaginary part is plotted on the $y$-axis. Each data point in this plot thus expresses a different frequency, starting with $0 \mathrm{~Hz}$ at the point $(x, 0)$. The length of a line that can be drawn between each point and $(0,0)$ corresponds to the magnitude of the admittance at a given frequency, whereas the angle between this line and the horizontal $x$-axis is the phase shift between the response and the stimulation. Therefore the units of both axes of complex admittance plots are nano-Siemens (nS). All transfer functions after electrode subtraction are shown as the more common impedance Bode plots.

Since no electrode resistance or capacitance compensation was used, the resulting voltage response (Figure 1C), and therefore the admittance transfer function, $Y(f)$, refers to both the electrode and the neuron. In the following all admittance transfer functions are written as $Y_{\mathrm{i}}$ with $\mathrm{i}$ being $\mathrm{e}, \mathrm{e}+\mathrm{n}$ or $\mathrm{e}+\mathrm{m}$ indicating that the electrode was measured alone, within a neuron or with a neuronal model, respectively.

During intracellular measurements, the properties of the electrode are best described as a frequency-, depth- (electrode tip with respect to the surface of the Ringer solution in the recording chamber), time- and current-dependent serial impedance, $Z_{\mathrm{ee}}(f, x$, $\left.t, I_{\mathrm{e}}\right)$, and a depth-dependent capacitance, $C_{\mathrm{e}}(x)$, parallel to the neuron. In addition to this parallel electrode capacitance, a constant parallel stray capacitance, $C_{s}$, (Figure 1A) is always present, i.e. the capacitance to ground at the input of the buffer operational amplifier (Molecular Devices, 2008). In order to obtain reliable data on frequency responses of single neurons recorded with sharp electrodes, a procedure called Piece-wise Non-linear Electrode Compensation (PNEC) was developed to remove the electrode properties from the total response in the frequency-domain. To test the PNEC method a passive neuronal circuit model composed of a thin film resistance with $R_{\mathrm{m}}=10 \mathrm{M} \Omega$ (Tolerance: $\pm 0.02 \%$ ) (Vishay Sfernice, Pennsylvania, USA) parallel to a polystyrene capacitor with $C_{\mathrm{m}}=1 \mathrm{nF}$ (Tolerance: $\pm 1 \%$ ) was used. For neuronal or model measurements the root-mean-square (RMS) error was computed separately for impedance magnitude and phase. The degree of electrode nonlinearity $(\lambda)$ was calculated as the slope of the linear regression between injected current and the steady state electrode resistance (Brette et al., 2008). Matlab (Mathworks Corp.) was used for computations. For time-domain simulations Simulink was used in combination with the SimPowerSystems Blockset to model the electrode and the passive neuron as electrical circuits. For AEC compensation the kernel computation and electrode kernel extraction was done using the latest Python implementation from http://audition.ens.fr/brette/HRCORTEX/AEC/AECcode.html. For linear regression calculations the Matlab function "regress" was used. A model for the electrode has been created by fitting two distributed capacitances and resistances (RC's) and a serial resistance and inductance (R-L) to the measured frequency response of an electrode using the Matlab function "lsqcurvefit". Graphical 
presentations were made with Corel Draw (Corel Corporation Ltd.). Statistica 6.1 was used for the Wilcoxon matched pairs test.

\section{RESULTS}

\section{ELECTRODE PROPERTIES}

Electrode properties were revealed by using a multi-sine analysis of the electrodes in the recording chamber and/or the tissue to determine the electrode transfer function, $Y_{\mathrm{e}}$. In contrast to a simple electrode model (constant parallel capacitance and resistance), which approximates a theoretically expected straight line on an admittance plot (red line in Figure 2A1), the glass microelectrodes used for the present intracellular recordings show a complex frequency-dependent behavior of the real part (green and blue lines in Figure 2A1) but an essentially linear behavior of the imaginary part, similar to a simple RC electrode model (Figure 2A2). This behavior depended on the depth $(x)$ of the electrode tip in the tissue and/or bath with respect to the level of the Ringer solution; in particular, the capacitance of the electrode increased with the depth (Figure 2A2). Even though the resistance of sharp electrodes, filled with $\mathrm{KCl}$ or $\mathrm{KAc}$, could fluctuate randomly within the range of minutes (Figure 2B1), the shape of the admittance measurements and its imaginary component (Figure 2B2) did not change over time. This spontaneous impedance change was considerably reduced, yet not absent when the electrode tip was inside a neuron. Injections of holding currents $I_{\text {hold }}$ through the electrode radically changed the real part of the admittance measurements, but had little effect on its imaginary part (Figures 2C1,C2). This behavior can be best seen in the corresponding impedance magnitude plot (Figure 2C1 inset). Also, note that some sharp electrodes showed a resonance $(*$ in Figure $2 \mathrm{Cl}$ inset) at $\sim 50 \mathrm{~Hz}$ that occurred mostly for positive $I_{\text {hold }}$ injections. Considering these results and taking into account the additional stray capacitance of the amplifier, the electrode transfer function can be described as

$Y_{\mathrm{e}}=\frac{1}{Z_{\mathrm{ee}}\left(f, x, t, I_{\mathrm{e}}\right)}+j 2 \pi f\left[C_{\mathrm{e}}(x)+C_{\mathrm{s}}\right]$

where $Z_{\mathrm{ee}}$ is the complex impedance of the electrode without an assumed passive capacitance. Thus, $\operatorname{real}\left(Y_{\mathrm{e}}\right)=\operatorname{real}\left[1 / Z_{\mathrm{ee}}\left(f, x, t, I_{\mathrm{e}}\right)\right]$ with an essentially frequency-, depth-, time- and currentdependency and imaginary $\left(Y_{\mathrm{e}}\right)=j 2 \pi f\left[C_{\mathrm{e}}(x)+C_{\mathrm{s}}\right]+$ imagina$\operatorname{ry}\left[1 / Z_{\mathrm{ee}}\left(f, x, t, I_{\mathrm{e}}\right)\right]$, with the latter being dominated by a depth $(x)$-dependent electrode capacitance, $C_{\mathrm{e}}(x)$, plus $C_{\mathrm{s}}$, the amplifier stray capacitance. Figures 2A2,B2,C2 shows that the imaginary part of $Y_{\mathrm{e}}$ depends linearly on the frequency and is thus almost completely described by $j 2 \pi f\left[C_{\mathrm{e}}(x)+C_{\mathrm{s}}\right]$. Therefore, the parallel capacitance, $C=C_{\mathrm{e}}+C_{\mathrm{s}}$ can be estimated by fitting the function $2 \pi f C$ to imaginary $\left(Y_{\mathrm{e}}\right)$ using a linear regression. For all electrodes in the present study, the regression was highly significant (all $\left.R^{2}>0.990\right)$.

\section{PIECE-WISE NON-LINEAR ELECTRODE COMPENSATION (PNEC) PROCEDURE}

Because of the particular complex electrode behavior, no electrode compensation (neither resistance nor capacitance compensation) was used, but a procedure called Piece-wise Non-linear Electrode Compensation (PNEC) was developed to subtract the electrode from the frequency-domain response offline after the measurements. The subtraction procedure involved measuring the electrode alone just after removing it from the neuron, but leaving it in the immediate vicinity $(5-8 \mu \mathrm{m})$ of the cell and applying the same holding currents, $I_{\text {hold }}$, as used during the intracellular recording. The latter procedure was necessary because of the current-dependent non-linearities of the electrode. As indicated above, changes in the electrode impedance observed either spontaneously or after impalement (Brette et al., 2008) are most likely due to partial blocking or unblocking of the micropipette tip as suggested by the finding that this behavior can be described by translating the real part of $Y_{\mathrm{e}}$ along the real axis, which is equivalent to adding a conductance $G_{\mathrm{T}}$ to the admittance $Y_{\mathrm{e}}$.

The impedance $Z_{\mathrm{n}}$ of a passive neuron (resistance $R_{\mathrm{n}}$ and capacitance $C_{\mathrm{n}}$ ) can be expressed by:

$Z_{\mathrm{n}}=\left(R_{\mathrm{n}}^{-1}+j 2 \pi f C_{\mathrm{n}}\right)^{-1}=\frac{R_{\mathrm{n}}-j 2 \pi f C_{\mathrm{n}} R_{\mathrm{n}}^{2}}{1+4 \pi^{2} f^{2} C_{\mathrm{n}}^{2} R_{\mathrm{n}}^{2}} \quad$ and $\lim _{f \rightarrow \infty} Z_{\mathrm{n}}=0$

with $\tau_{\mathrm{n}}=R_{\mathrm{n}} C_{\mathrm{n}}$

$\operatorname{real}\left(Z_{\mathrm{n}}\right)=\frac{R_{\mathrm{n}}}{1+4 \pi^{2} f^{2} \tau_{\mathrm{n}}^{2}}$

Thus at high frequencies, the neuron is shunted by its capacitance such that the electrode + neuron admittance measurement $Y_{\mathrm{e}+\mathrm{n}}$ should overlap with the electrode measurement $Y_{\mathrm{e}}$. Therefore, in order to compensate for the change in electrode impedance after leaving the neuron, $Y_{\mathrm{e}}$ was translated along the real axis until it superimposed on $Y_{\mathrm{e}+\mathrm{n}}$ at high frequencies above $f_{\mathrm{fit}}$. For $f_{\text {fit }}$, the frequency was chosen where the resistance of the neuron reaches $1 \%$ of its baseline resistance. Knowledge of the membrane time constant $\left(\tau_{\mathrm{n}}\right)$ of a typical recorded neuron, $f_{\text {fit }}$ can thus be calculated by:

$f_{\text {fit }}=\sqrt{\frac{\frac{1}{0.01}-1}{4 \pi^{2} \tau_{\mathrm{n}}^{2}}}$

A $\tau_{\mathrm{n}}=2 \mathrm{~ms}$, as measured in e.g. tonic and phasic $2^{\circ} \mathrm{VN}$ (Straka et al., 2004) gives a $f_{\text {fit }}$ of $792 \mathrm{~Hz}$, which was used in our experiments. With a typical neuronal resistance of $10 \mathrm{M} \Omega$ and typical electrode resistances of $\sim 100 \mathrm{M} \Omega$ the theoretical error calculated for the electrode resistance estimation is thus only $\sim 0.1 \%$ in our experiments.

After translating and determining the conductance $G_{\mathrm{T}}$, the electrode admittance measurements can be used to remove the electrode. Thus, this electrode compensation procedure, referred to as PNEC, is as follows:

(1) To estimate the conductance $G_{\mathrm{T}}$ the error $\left[\operatorname{real}\left(G_{\mathrm{T}}+Y_{\mathrm{e}}\right)-\right.$ $\left.\operatorname{real}\left(Y_{\mathrm{e}+\mathrm{n}}\right)\right]^{2}$ has to be minimized in the frequency range between $f_{\text {fit }}$ and the maximal frequency $\left(f_{\max }\right)$ measured. Since $G_{\mathrm{T}}$ is real, the estimation of $G_{\mathrm{T}}$ simplifies to:

$G_{\mathrm{T}}=\operatorname{mean}\left[\operatorname{real}\left(Y_{\mathrm{e}+\mathrm{n}}\right)-\operatorname{real}\left(Y_{\mathrm{e}}\right)\right]$

(2) Fit $2 \pi f C$ to imaginary $\left(Y_{\mathrm{e}}\right)$, using linear regression, in order to remove the parallel electrode capacitance $C_{\mathrm{e}}(x)$, and amplifier stray capacitance $C_{s}$ :

$$
Y_{\mathrm{e}+\mathrm{n}-\mathrm{c}}=Y_{\mathrm{e}+\mathrm{n}}-j 2 \pi f C
$$



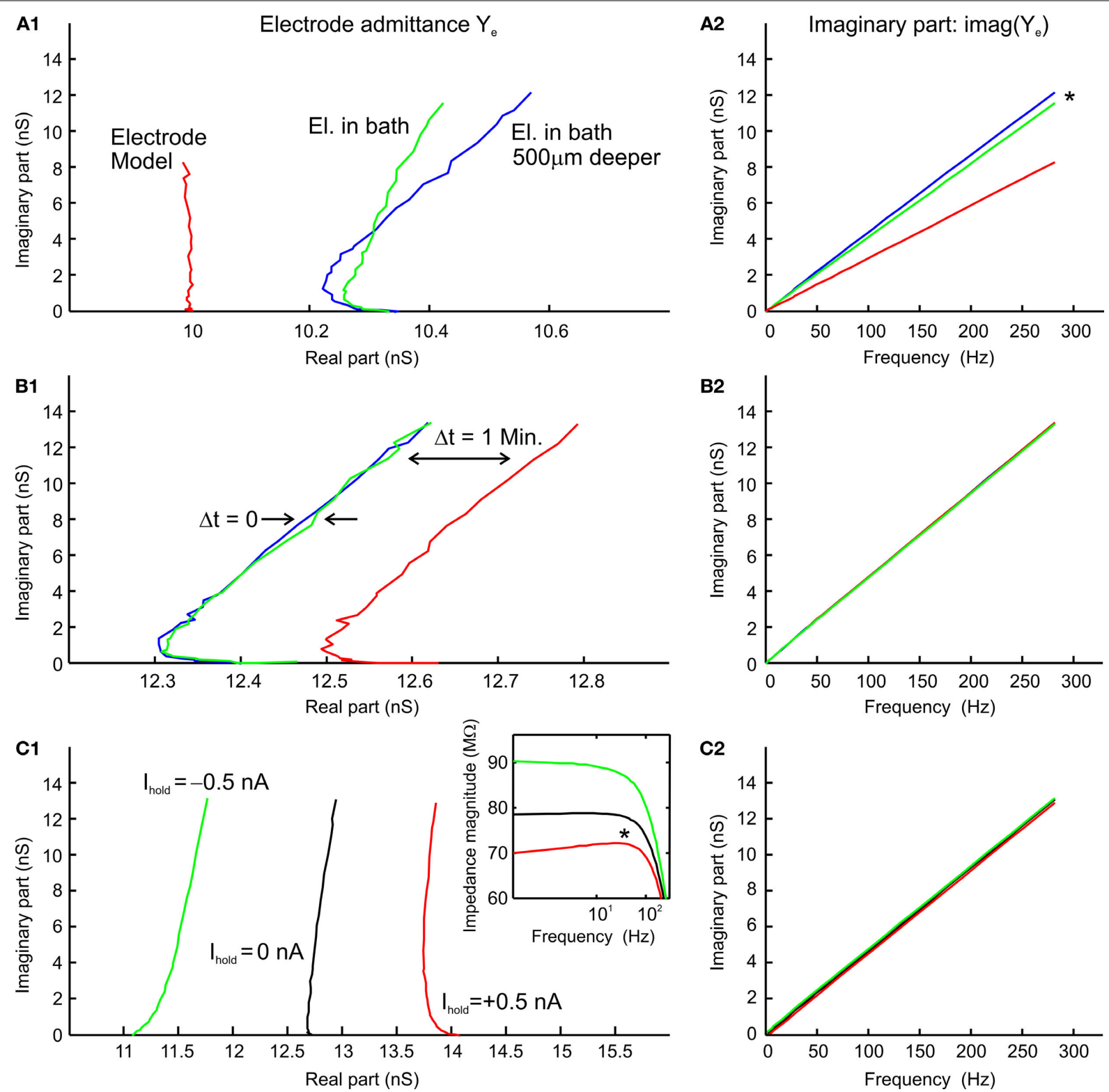

FIGURE 2 | Multi-sine analysis of electrode properties with discrete frequencies plotted for the range from $\mathbf{0 . 2}-300 \mathrm{~Hz}$. (A1,A2) Admittance plots (A1) of the electrode model circuit with constant parallel capacitance and resistance (red line); admittance plots (A1) of $\mathrm{KCl}(3 \mathrm{M})$ filled electrode with the tip at $1.5 \mathrm{~mm}$ (green line) and $2 \mathrm{~mm}$ below the surface of the Ringer solution (blue line) show frequency-dependent changes of electrode properties influenced by the depth in the Ringer solution; plots of the imaginary part (A2) show that the electrode capacitance increases with increasing depth of the electrode tip in the bath $\left({ }^{*}\right) ; I_{\text {hold }}=0$ nA. (B1,B2) Admittance plots (B1) of three measurements of electrode properties in the recording chamber; measurements 1 and 2 were consecutive (green and blue lines), whereas the measurement 3 (red line) was taken 1 min later; note that the admittance fluctuates over time while the capacitance (B2) remains stable; $I_{\text {tod }}=0 \mathrm{nA}$. (C1, C2) Admittance plots (C1) of three electrode measurements with different holding currents $I_{\text {hold }}=0 \mathrm{nA}$ (black line), $-0.5 \mathrm{nA}$ (green line), $+0.5 \mathrm{nA}$ (red line); injection of a constant current changes the real part of the electrode admittance response while the capacitance (C2) remains unchanged.
(3) Remove the serial impedance $Z_{\mathrm{ee}}\left(f, x, t, I_{\mathrm{e}}\right)=\left(G_{\mathrm{T}}+Y_{\mathrm{e}}-j 2 \pi f C\right)^{-1}$ of the electrode:

$$
Y_{\mathrm{n}}=\left[Y_{\mathrm{e}+\mathrm{n}-\mathrm{c}}^{-1}-\left(G_{\mathrm{T}}+Y_{\mathrm{e}}-j 2 \pi f C\right)^{-1}\right]^{-1}
$$

The PNEC procedure has been performed with 19 neurons: the 19 electrodes showed a mean resistance of $85.5 \pm 24.5 \mathrm{M} \Omega$ (mean \pm SD) and a mean capacitance of $8.16 \pm 2.01 \mathrm{pF}$; for $G_{\mathrm{T}}$ an average of $0.76 \pm 1.2 \mathrm{nS}$ (mean $\pm \mathrm{SD}$ ) was estimated, which resulted in an RMS of $0.0375 \pm 0.018 \mathrm{nS}$ (mean $\pm \mathrm{SD}$; from $f_{\text {fit }}$ to $\left.f_{\max }\right)$. The mean degree of nonlinearity $(\lambda)$ could be evaluated in 12 electrodes and resulted in $-2.56 \pm 3.9 \mathrm{M} \Omega / \mathrm{nA}($ mean $\pm \mathrm{SD})$ which is comparable to nonlinearities found previously (Brette et al., 2008). 


\section{MODEL TEST OF THE SUBTRACTION PROCEDURE Measuring a passive neuronal circuit model}

The PNEC procedure was tested by measuring a passive neuronal circuit model $\left(R_{\mathrm{m}}=10 \mathrm{M} \Omega, C_{\mathrm{m}}=1 \mathrm{nF}\right)$, attached to the electrical path-to-ground, through a real electrode in the bath (Figure 3A). Although this reproduces the serial resistive connection of an electrode to a real neuron, the electrode capacitance $C_{\mathrm{e}}$ is also serial to the neuron. Since the capacitance $C_{\mathrm{e}}$ is only parallel to the electrode it shunts only the electrode impedance, comparable to the parallel RC of neurons. Accordingly, at high frequencies the two measurements of $Y_{\mathrm{e}}$ and $Y_{\mathrm{e}+\mathrm{m}}$ do not overlap and the translation conductance $G_{\mathrm{T}}$ cannot be estimated. Thus $G_{\mathrm{T}}$ was chosen for this model test in such a way that the resistance of the model neuron $(10 \mathrm{M} \Omega)$ was correct at the lowest frequency after electrode subtraction. Figure 3B shows the admittance plots with $I_{\text {hold }}=1 \mathrm{nA}$ of electrode + model neuron $\left(Y_{\mathrm{e}+\mathrm{m}}\right)$ and the measured, but translated electrode $\left(G_{\mathrm{T}}+Y_{\mathrm{e}}\right)$. Figures 3C1,C2 illustrates that the impedance magnitude obtained after the PNEC procedure (orange lines) is in good agreement with the analytical values of the neuronal circuit model (green lines) (RMS: $0.22 \mathrm{M} \Omega$ ), but shows an error for the phase at high frequencies (RMS: $21.5^{\circ}$ ) that will be corrected below by taking into account the complete serial connection of the electrode to the test model system. Thus, both the lack of overlapping $Y_{\mathrm{e}}$ and $Y_{\mathrm{e}+\mathrm{m}}$ at high frequencies and the phase error are due to the serial connection of the electrode capacitance to the model. Since only the amplifier stray capacitance $C_{\mathrm{s}}$ is in parallel to the neuron model, its subtraction must be adapted to the test setup. This involves two individual subtractions of $C_{\mathrm{s}}=4.4 \mathrm{pF}$ that was estimated before by measuring a precision resistance, as follows:

$Y_{\mathrm{m}}=\left[\left(Y_{\mathrm{e}+\mathrm{m}}-j 2 \pi f C_{\mathrm{s}}\right)^{-1}-\left(G_{\mathrm{T}}+Y_{\mathrm{e}}-j 2 \pi f C_{\mathrm{s}}\right)^{-1}\right]^{-1}$

With this serial-PNEC the phase errors can be eliminated and the obtained impedance magnitude and phase plots (Figures $\mathbf{3 C 1}, \mathbf{C 2}$; black lines) match well with the analytical values for the neuronal circuit model (green lines) (RMS: $0.20 \mathrm{M} \Omega, 3.7^{\circ}$ ). This successful subtraction of electrode properties in the model test setup confirms that non-linear electrode properties can be appropriately taken into account during piece-wise linear measurements.

The critical need for correcting microelectrode measurements with measured electrode properties are illustrated by comparing the errors that result from assuming a simple RC electrode $\left(Y_{\mathrm{es}}\right.$ in Figure 3B) although it has a resistance identical to the low-frequency $(0.2 \mathrm{~Hz})$ impedance of the real electrode and an identical capacitance. Figure 3C shows that the serial subtraction, using adapted $C_{s}$ subtraction as above, of a simple RC electrode (red lines) results in a considerable error and even suggests an incorrect resonance behavior because the real electrode has not been correctly taken into account (RMS: $3.40 \mathrm{M} \Omega, 41^{\circ}$ ). To further quantify the quality of fit, the correlations between the residuals (estimated magnitude and phase minus theoretical values) and the frequency have been analyzed for all three electrode subtractions. The residual error after BC or PNEC still showed a correlation with frequency for both magnitude and phase, but there was no significant correlation using serial-PNEC.

Similar errors using simple RC electrode compensation can also be seen in the data from recorded neurons. Using the PNEC procedure for a frog phasic $2^{\circ} \mathrm{VN}\left(I_{\text {hold }}=0 \mathrm{nA}\right.$; Figures 3D1,D2) the magnitude and phase plots (black lines) suggest rather passive membrane properties. However, an incorrect subtraction using a simple RC electrode, with a resistance identical to the low-frequency impedance and capacitance identical to the real electrode, yields a considerable resonance (red lines) that however is an artifact due to the incorrect electrode compensation.

\section{Comparison of PNEC, BC and AEC using an electrode model}

Using a time-domain simulation in Matlab Simulink the performance of PNEC was compared to Bridge- (BC) and Active Electrode Compensation (AEC, Brette et al., 2008). The frequency-domain behavior of a typical electrode (Figure 3E1 gray plot) was modeled by using distributed capacitances and resistances (RC's) reflecting the rightward bend in the electrode admittance plots at high frequencies and a serial resistance and inductance (R-L) in parallel to the last distributed RC to account for the resonant behavior at low frequencies. Thus the prominent electrode behaviors could be fitted ( $Y$ in Figure 3E1 black line) using two distributed RC's of 14.5 or $55.5 \mathrm{M} \Omega$ and 4.9 or $2.8 \mathrm{pF}$, respectively and a R-L with $2.2 \mathrm{G} \Omega$ and $100 \mathrm{MH}$ (Figure 3E1 inset). To mimic the electrode resistance shift for the electrode inside the neuron, the resistance of the last $\mathrm{RC}$ was reduced from $55.5 \mathrm{M} \Omega$ to $53.5 \mathrm{M} \Omega$ when a purely passive RC-type neuron was added which, together with the electrode, produced the admittance response $Y_{\mathrm{e}+\mathrm{m}}$ as shown exemplarily for a low resistance neuron model (low-R: $R_{\mathrm{n}}=10 \mathrm{M} \Omega, C_{\mathrm{n}}=200 \mathrm{pF}$ ) in Figure 3E1 (green line).

For the simulation of $\mathrm{BC}$, capacitance and resistance compensation have been directly implemented by simulating standard compensatory circuits (Molecular Devices, 2008) in Simulink: resistance compensation has been set to the low-frequency resistance of the electrode and capacitance compensation has been used maximally just before ringing occurred. For AEC, only capacitance compensation has been simulated in Simulink and the resistance has been subtracted offline using the provided AEC procedure (Brette et al., 2008). Prior to this simulation, the correct implementation of AEC could be confirmed using an electrode and neuron model as used in the simulations of Brette et al. (2008; not shown).

The simulation has been realized for two types of neurons having a time constant of 2 ms: a low resistance neuron (low-R: $R_{\mathrm{n}}=10 \mathrm{M} \Omega, C_{\mathrm{n}}=200 \mathrm{pF}$, solid lines) and a high resistance neuron (high-R: $R_{\mathrm{n}}=100 \mathrm{M} \Omega, C_{\mathrm{n}}=20 \mathrm{pF}$, dotted lines), mimicking a large and small cell, respectively. Application of the PNEC procedure (with $f_{\text {fit }}=792 \mathrm{~Hz}$ ), i.e. shifting $G_{\mathrm{T}}+Y_{\mathrm{e}}$ (Figure 3E1 dotted line) and subtracting the electrode admittance, results in only small errors (RMS: low-R: $0.2 \mathrm{M} \Omega, 3.4^{\circ}$; high-R: $1.6 \mathrm{M} \Omega, 3.5^{\circ}$ ) for the impedance magnitude and phase plots (black solid or dotted lines) when compared to the expected analytical results of the passive neuron (green solid or dotted lines) (Figures 3E2,E3). In contrast, using BC (Figures 3E2,E3 red solid or dotted lines) magnitude and phase errors (RMS: low-R: 10.5 M $2,20.1^{\circ}$; high-R: $7.6 \mathrm{M} \Omega, 21.0^{\circ}$ ) occur due to incorrect electrode compensation. For AEC (blue solid or dotted lines) (RMS: low-R: $0.8 \mathrm{M} \Omega, 6.7^{\circ}$; high-R: $5.5 \mathrm{M} \Omega, 7.2^{\circ}$ ), errors already occur at the low-frequency end due to the underestimation of the electrode resistance especially with the high resistance neuron, which results from the low ratio of electrode and membrane time constants. In addition, the inductive component of the electrode further complicates the correct electrode kernel 
A

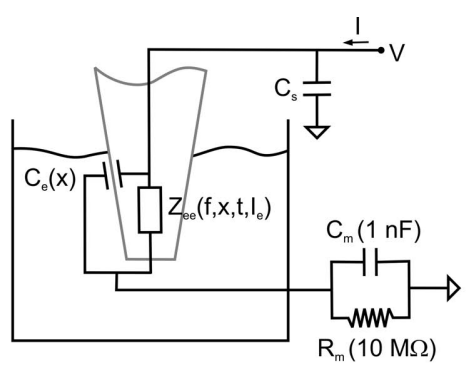

C1

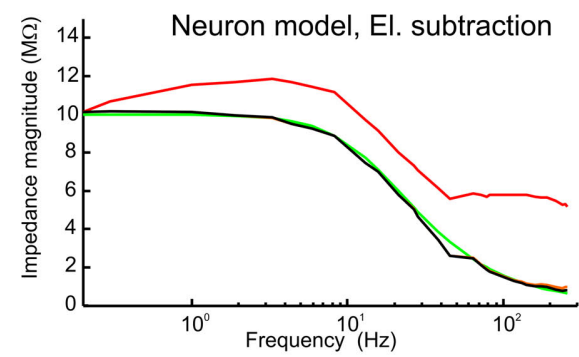

D1

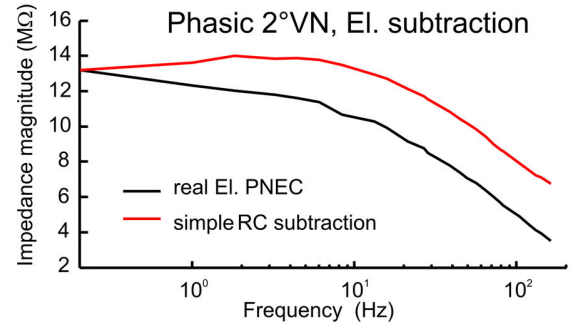

E1

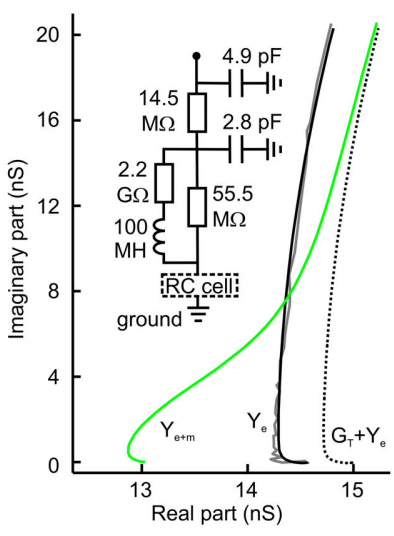

E2

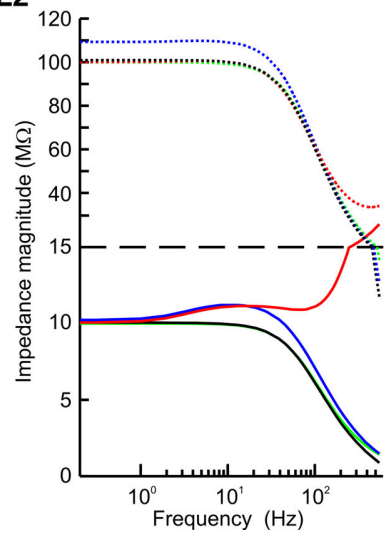

B 30 Neuron model, $\mathrm{I}_{\text {hold }}=1 \mathrm{nA}$

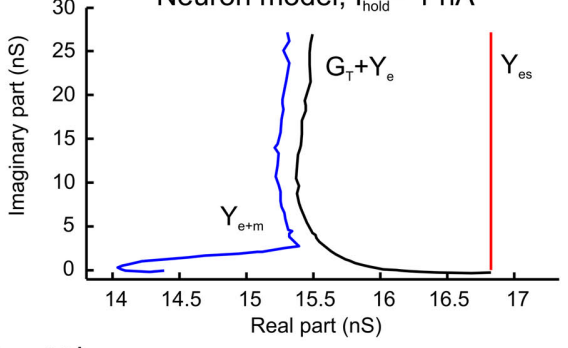

C2

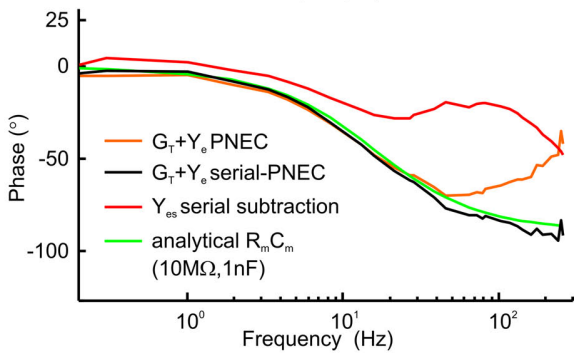

D2

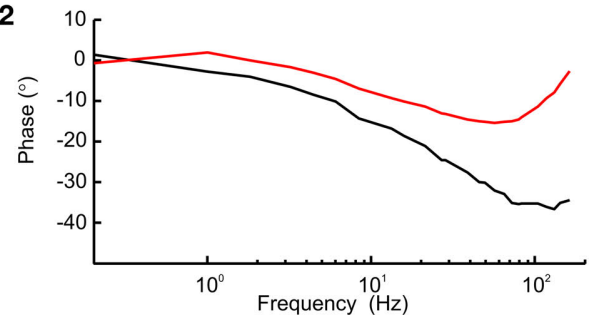

E3

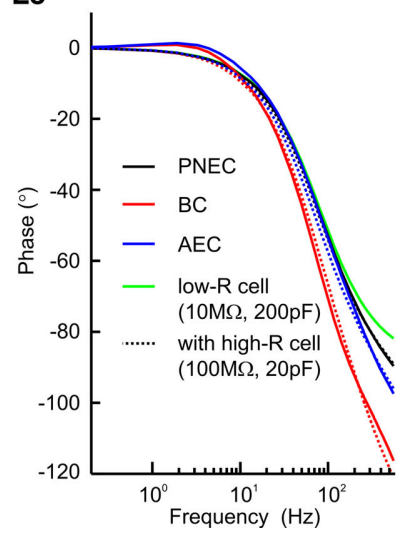

\section{FIGURE 3 | Model test of the Piece-wise Non-linear Electrode}

Compensation (PNEC) method. (A) Equivalent circuit diagram of the test setup with the electrode in the Ringer-filled recording chamber representing a parallel impedance $Z_{e \mathrm{e}}\left(f, x, t, I_{\mathrm{e}}\right)$ and a capacitance, $C_{\mathrm{e}}(x)$, serial to a passive neuronal circuit model $\left(R_{\mathrm{m}}=10 \mathrm{M} \Omega, C_{\mathrm{m}}=1 \mathrm{nF}\right)$ attached to the electrical pathto-ground and a constant amplifier stray capacitance, $C_{s^{\prime}}$ parallel to the model. (B) Admittance plots of the corrected electrode alone $G_{T}+Y_{\mathrm{e}}$ (black line) of the electrode with an attached neuronal circuit model $Y_{e+m}$ (blue line) and the analytical response of a simple RC-type electrode model $Y_{\text {es }}$ (red line); $I_{\text {hold }}=+1 \mathrm{nA}$, plotted frequency spectrum $0.2-485 \mathrm{~Hz}$. (C1, C2) Bode plots of a passive neuronal circuit model using different electrode compensation methods on the combined measurement $Y_{e+m}$ : (a) electrode $G_{T}+Y_{e}$ (orange lines) with PNEC, (b) electrode $G_{T}+Y_{e}$ using serial-PNEC (black lines), (c) simple theoretical $R C$ electrode, $Y_{\text {es' }}$ using serial subtraction (red lines) and (d) the analytical passive neuronal circuit model (green lines). Note that using the simple RC electrode compensation for the passive neuronal model shows a resonance that is solely an electrode compensation artifact; trace labels in (C2) also apply to (C1). (D1,D2) Results from the subtraction of a simple RC-type electrode (red lines) and using PNEC with the actual electrode (black lines) on the intracellular records of a phasic $2^{\circ} \mathrm{VN}$; note that subtraction of a simple RC-type electrode also shows an incorrect resonance for the actual neuron that is caused by the above electrode artifact; trace labels in (D1) apply also to (D2). (E1) Timedomain simulation of PNEC, AEC and BC; admittance plots of an electrode with distributed capacities and parallel resistance and inductance (inset) $Y_{e}$ (black line) with parameters adjusted to mimic the behavior as measured in real electrodes (gray line), with an attached passive RC neuronal circuit model (10 M $2,200 \mathrm{pF}$ ) and reduced overall resistance $Y_{\mathrm{e}+\mathrm{m}}$ (green line) and shifted electrode $G_{\mathrm{T}}+Y_{\mathrm{e}+\mathrm{m}}$ (dotted line) prior to PNEC subtraction; plotted frequency spectrum 0-556.2 Hz. (E2,E3) Results after electrode compensation with a low resistance $R C$ cell (low-R: $10 \mathrm{M} \Omega, 200 \mathrm{pF}$ ) (solid lines) or a high resistance RC cell (high-R: $100 \mathrm{M} \Omega$, $20 \mathrm{pF}$ ) (dotted lines): simple BC (red) or AEC compensation (blue) causes electrode resonance artifacts in the impedance (E2) and phase (E3), while PNEC (black) complies well with the expected results (green); change of $y$-coordinate scale at $15 \mathrm{M} \Omega$ (dashed line) in (E2); trace labels in (E3) also apply to (E2). 
estimation and results in a resonance that is an artifact due to the incorrect electrode compensation, which particularly can be seen in the low-R simulation. The simulations further show that the electrode compensation error in AEC is not solely dependent on the time constant ratio, as stated by Brette et al. (2008) but also increases with the resistance of the cell. This is also the case for PNEC, but here the error can be further reduced by increasing $f_{\text {fit }}$.

This comparison shows that the PNEC method is well suited to compensate for the complicated electrode behavior and yields better results compared to $\mathrm{BC}$ and even AEC. However, PNEC is also not perfect, since minor errors occur for the phase at very high frequencies, likely due to an insufficient compensation of distributed electrode capacitances. This suggests that PNEC is reliable at least up to $\sim 300 \mathrm{~Hz}$ for the estimation of neuronal frequency-domain transfer functions.

\section{MEASUREMENT RESULTS}

Examples of electrode compensation for three different neuronal subtypes recorded with different holding currents $\left(I_{\text {hold }}\right)$ in an isolated adult frog whole brain were used to illustrate the applicability of PNEC for typical neuronal recordings. Figure 4A illustrates the electrode adjustment procedure for an AbMot at $I_{\text {hold }}=+1 \mathrm{nA}$, which required a shift in the electrode admittance response, $Y_{\mathrm{e}}$, due to a difference in the electrode properties when it was removed from the neuron. As described above, the abscissa values were shifted prior to the subtraction in order for the curves to overlap at high frequencies above $f_{\text {fit }}$ indicated as $G_{\mathrm{T}}+Y_{\mathrm{e}}$. Electrode corrected magnitude and phase plots for this AbMot at other holding currents illustrate the marked potential dependence of the neuronal impedance including a pronounced resonance (Figures 4 B1,B2). Bode plot examples for previously described tonic (Figures 4C1,C2) and phasic (Figures 4D1,D2,E1,E2) $2^{\circ} \mathrm{VN}$ (Beraneck et al., 2007) indicate that the membrane resonance properties reported for phasic $2^{\circ} \mathrm{VN}(*$ in Figures 4D1,E1) are not an electrode artifact.

Multi-sine analysis allows direct visualization of the dynamic responses of functionally different neuronal phenotypes. For instance, the AbMot, which is plotted in Figures 4B1,B2 revealed a high degree of resonance during depolarizing currents, most likely caused by an interaction of delayed rectifier potassium and sodium channels (Hutcheon and Yarom, 2000). Frog tonic $2^{\circ} \mathrm{VN}$ have no membrane resonance behavior but exhibited an increasing impedance with membrane depolarization (Figures 4C1,C2). This latter effect is likely caused by non-inactivating calcium or sodium inward currents as suggested previously (Beraneck et al., 2007). In contrast, frog phasic $2^{\circ} \mathrm{VN}$ showed decreasing impedance and increasing resonance $\left({ }^{*}\right.$ in Figures 4D1,E1) with membrane depolarization due to the activation of low-threshold voltage-dependent $I_{\mathrm{D}}$-type potassium channels as reported earlier (Beraneck et al., 2007). Furthermore, since $I_{D}$-type potassium channels are specifically blocked by low concentrations of 4-AP (Wu et al., 2001), bath application of $20 \mu \mathrm{M} 4$-AP during multi-sine measurements of a phasic $2^{\circ} \mathrm{VN}$ (dotted lines in Figures 4D1,D2) leads to an increase in impedance magnitude for $I_{\text {hold }}=0$ and $I_{\text {hold }}=0.5 \mathrm{nA}$.

To demonstrate the errors induced by insufficient bridge compensation (BC), another phasic $2^{\circ} \mathrm{VN}$ (Figures 4E1,E2) has been additionally measured during standard $\mathrm{BC}$ from the amplifier (dotted lines). While both compensation types match fairly well at the lowest frequencies for $I_{\text {hold }}=0$ and $-0.5 \mathrm{nA}$, the measurements at depolarized membrane potentials show an overcompensation due to electrode rectification. Furthermore incorrect compensation of the distributed electrode RCs in BC leads to bends in the magnitude and large jumps in the phase at higher frequencies $(\sim 200 \mathrm{~Hz})$.

To compare the PNEC method with standard bridge compensation (BC), both techniques have been applied in a total of 11 neurons at $I_{\text {hold }}=0 \mathrm{nA}$. Since the measurements were made at resting membrane potential, these neurons presumably behave like passive RC circuits with an impedance of $Y_{n}=G_{n}+j 2 \pi f C_{n}$. Thus $C_{n}$ can be estimated by fitting $j 2 \pi f C_{\mathrm{n}}$ to imaginary $(Y)$ using a linear regression and $G_{\mathrm{n}}=$ mean $[\operatorname{real}(Y)]$, where $Y$ is the measured neuronal admittance after either PNEC or BC compensation. The root-meansquare error of this fit is calculated as RMS $=\sqrt{\operatorname{mean}\left(\left|Y_{n}-Y\right|^{2}\right)}$. This test resulted in a better RMS for PNEC, median of RMS: $0.0458 \mu \mathrm{S}$, compared to BC, median of RMS: $0.1818 \mu \mathrm{S}$ (difference highly significant with $p=0.0076$, Wilcoxon matched pairs test).

\section{TEST OF PIECE-WISE LINEARITY}

Control experiments were performed to evaluate the validity of the assumption of piece-wise linearity for PNEC. Measurements of the electrode alone with different multi-sine half-amplitudes $\left(h_{\text {Amp }}=\right.$ half of peak-to-peak amplitude of multi-sine stimulus; $h_{\text {Amp }}=1 \mathrm{nA}$ and $h_{\text {Amp }}=0.5 \mathrm{nA}$ ) showed that, apart from the time-dependent translational shift of the electrode, the frequency response itself (e.g. $I_{\text {hold }}=+0.5 \mathrm{nA}$ in Figure 5A) does not change with increasing amplitude. Nevertheless, as shown before, frequency responses are significantly affected by the holding $I_{\text {hold }}$ current level (compare with Brette et al., 2008). A further indication of the linearity over the range of multi-sine amplitudes is the successful test subtraction procedure (Figures $\mathbf{3 A - C 2}$ ). Thus, this is a piece-wise linear analysis of two non-linear elements, the neuron and the electrode, both of which show marked non-linearities at different holding current levels. The non-linear effects of the neuron itself are due to its voltage-dependent ion channels. Measuring AbMot with an $I_{\text {hold }}=0 \mathrm{nA}$ and multi-sine currents with three different half-amplitudes of $h_{\mathrm{Amp}}=0.4 \mathrm{nA}, 0.6 \mathrm{nA}$ and $0.8 \mathrm{nA}$ showed that, apart from some fluctuations in the phase using the largest multi-sine current $\left(h_{\mathrm{Amp}}=0.8 \mathrm{nA}\right)$, all measurements yield identical results (Figures 5B1,B2). The source of these phase fluctuations using $h_{\text {Amp }}=0.8 \mathrm{nA}$ could be pinpointed by the observation of $V_{\text {AveM }}$, which indicated that the multi-sine current triggered a few spikes (not shown). This result illustrates an important restriction required for most linearization methods, namely that the multisine amplitude should be chosen such that it is small enough not to trigger action potentials, but large enough to have a reasonable signal-to-noise ratio.

\section{OUTLOOK: COMPENSATION IN TIME-DOMAIN MEASUREMENTS}

Since we are considering piece-wise linear systems, the frequencydomain data can be translated into the time-domain, and therefore it is also possible to use the present PNEC framework to dissociate electrode from neuronal responses for arbitrary stimuli, as long as the current amplitude of the latter remain in the linear range. In the following, a method is presented that allows the determination of the electrode kernel for electrode compensation in the timedomain using the described PNEC framework. For this purpose, 

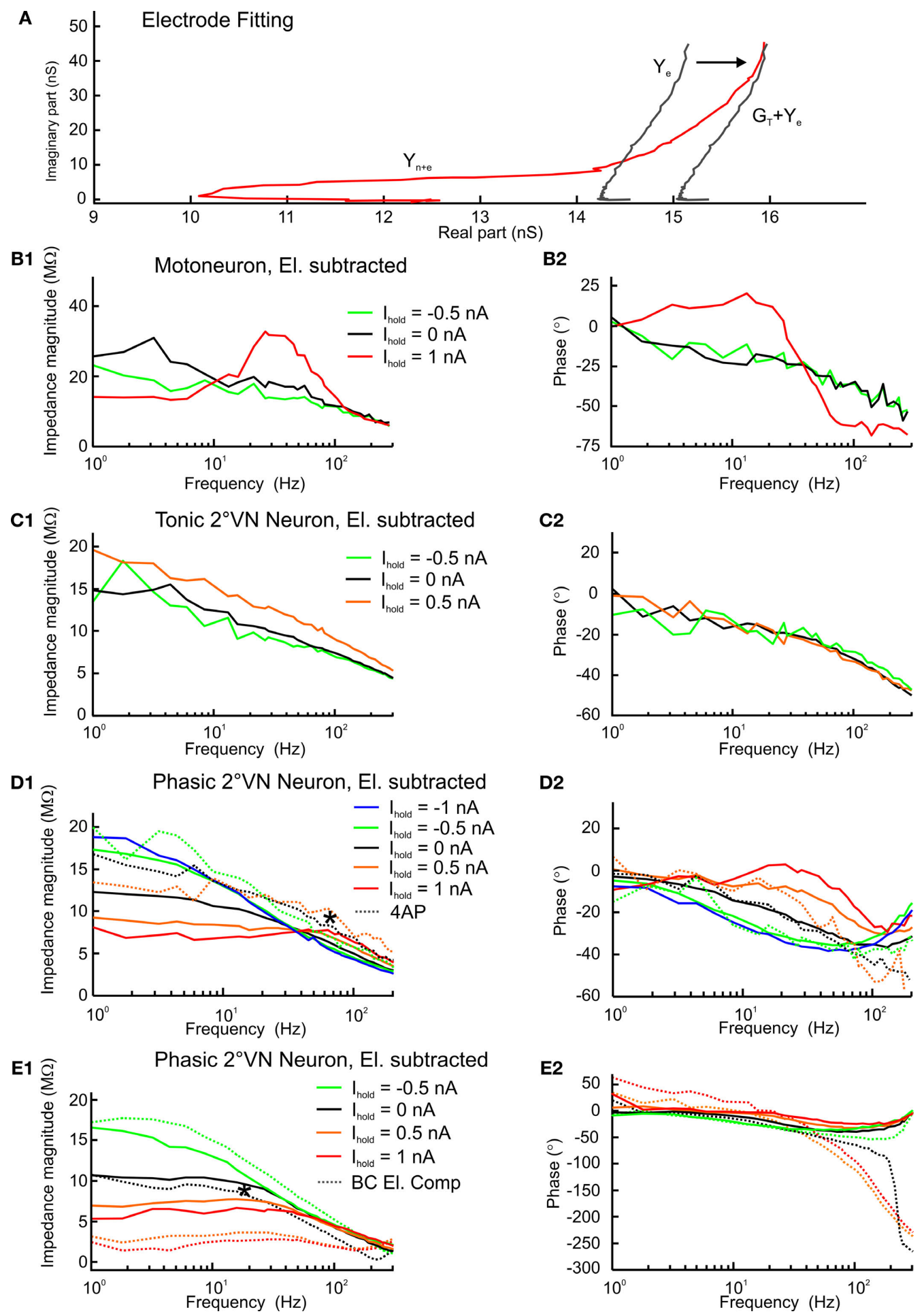

FIGURE 4 | Electrode fitting and response dynamics of second-order vestibular neurons $\left(2^{\circ} \mathrm{VN}\right)$ and abducens motoneuron (AbMot) using Piece-wise Non-linear Electrode Compensation (PNEC). (A) Electrode fitting procedure of the responses in an AbMot at $I_{\text {hold }}=+1 \mathrm{nA}$; the electrode admittance $Y_{e}$ (solid line) changed after the electrode was removed from the neuron and was shifted $\left(G_{T}+Y_{\mathrm{e}}\right)$ in order to overlap at high frequencies with the properties of the electrode + neuron admittance $Y_{n+e^{\prime}}$; plotted frequency spectrum 0.2-980 Hz. (B1,B2-D1,D2) Impedance profiles and corresponding phase relations of the responses at several holding currents (I ) of an AbMot (B1,B2), a tonic $(\mathbf{C 1}, \mathbf{C 2})$ and two different phasic $2^{\circ} \mathrm{VN}(\mathbf{D 1}, \mathbf{D 2}, \mathbf{E 1}, \mathbf{E 2})$ after $\mathrm{PNEC}$; responses of the phasic $2^{\circ} \mathrm{VN}$ shown in $(\mathbf{D} 1, \mathbf{D 2})$ were measured before and after bath application of 4-aminopyridine (4AP; $20 \mu \mathrm{M})$ [dotted lines in (D1,D2)] with PNEC; responses of the phasic $2^{\circ} \mathrm{VN}$ shown in $(\mathbf{E 1 , E 2 )}$ were measured after PNEC as well as during standard bridge compensation (BC) of the amplifier [dotted lines in (E1,E2)]; legends in (B1-E1) also apply to (B2-E2) 


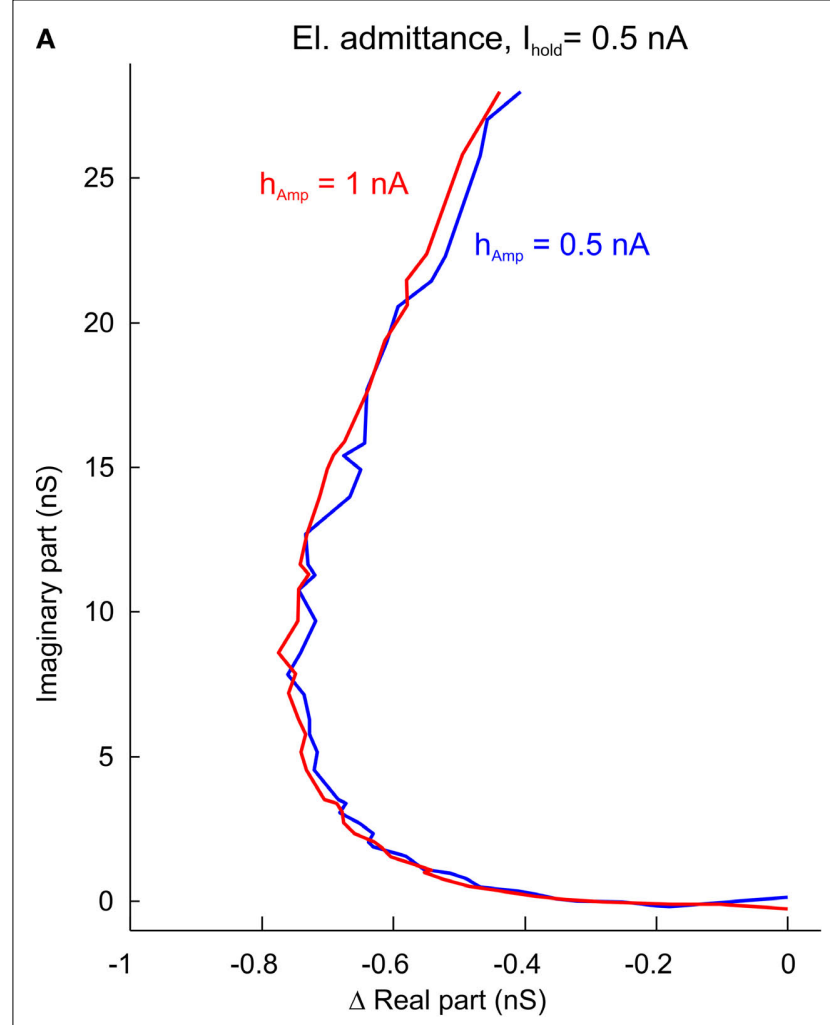

C1

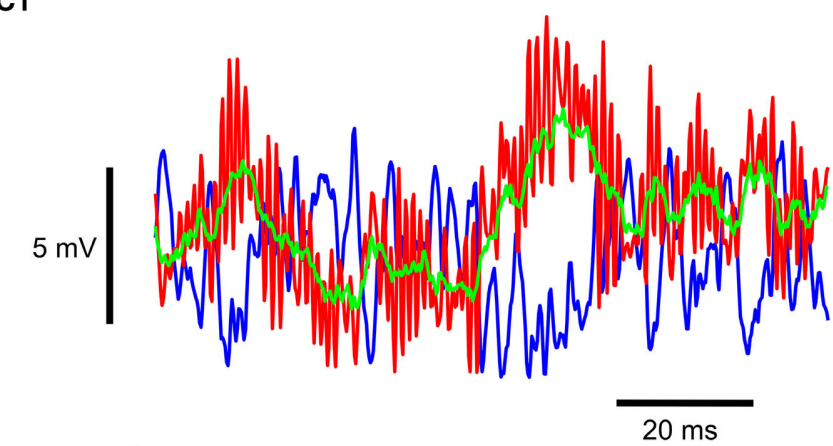

$0.5 \mathrm{nA}$

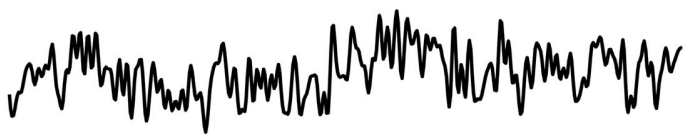

FIGURE 5 | Test of piece-wise linearity and offline compensation in time-domain measurements. (A) Admittance plot of the electrode properties at half peak-to-peak multi-sine current amplitudes of $h_{\text {Amp }}=0.5 \mathrm{nA}$ (blue line) and $h_{\text {Amp }}=1 \mathrm{nA}$ (red line); real part is shown as relative conductance to correct for the temporal fluctuation; plotted frequency spectrum $0.2-625 \mathrm{~Hz}$.

(B1,B2) Bode plots of the impedance profiles of an AbMot at $I_{\text {hold }}=0 \mathrm{nA}$ and peak-to-peak multi-sine current half amplitudes of $0.4 \mathrm{nA}$ (blue lines), $0.6 \mathrm{nA}$
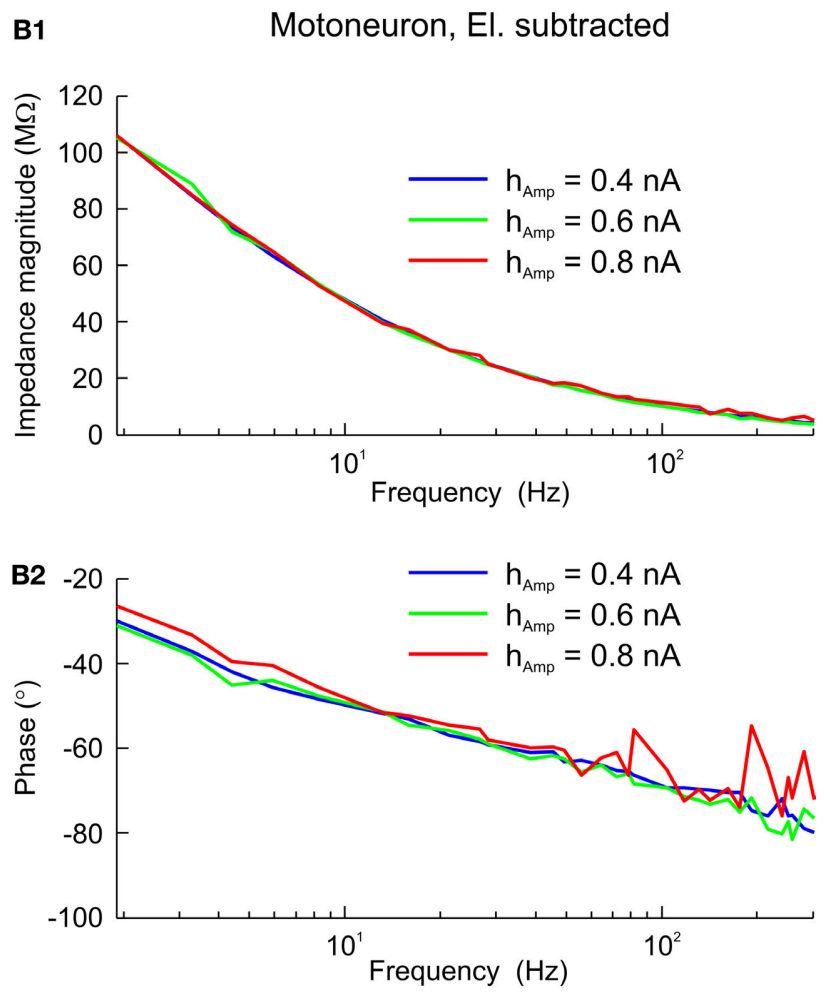

C2

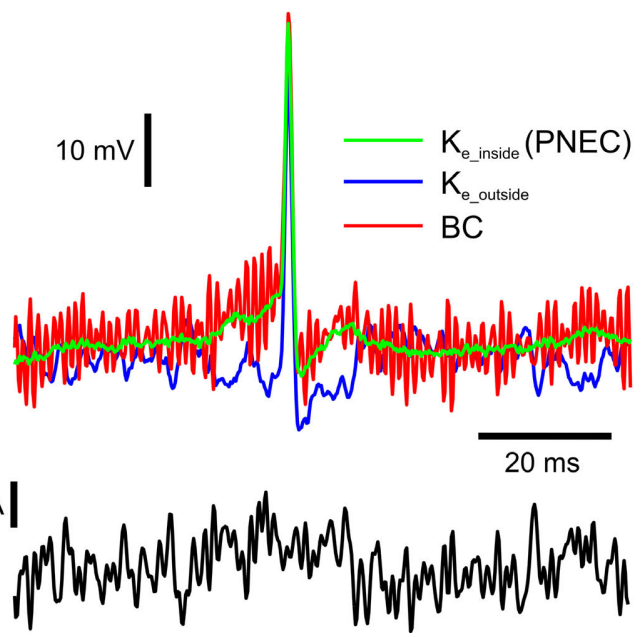

(green lines) and $0.8 \mathrm{nA}$ (red lines) after using Piece-wise Non-linear Electrode Compensation (PNEC). (C1,C2) Time-domain off-line subtraction of electrode voltage responses in a phasic (C1) and a tonic $2^{\circ} \mathrm{VN}$ (C2) during multi-sine stimulation (black traces) using the PNEC procedure for kernel determination (green traces), simple constant resistance/bridge compensation (red traces) and uncorrected electrode kernels $K_{\text {e_outside }}$ measured outside the cell (blue traces). multi-sine measurements of the electrode alone, outside the cell, were used to calculate the electrode kernel $K_{\text {e_outside }}$ via crosscorrelation between input current and output voltage (similar to Brette et al., 2008). These electrode kernels however differ from the real electrode kernel inside the cell and were thus corrected using the conductance $G_{\mathrm{T}}$, estimated via the second measurement inside the cell, as described before. For this correction, the kernel $K_{\mathrm{e} \text { outside }}$ was transformed into the frequency domain via FFT, shifted by $G_{\mathrm{T}}$, which was estimated using the first step of PNEC, and transformed back into the time-domain using an inverse FFT:

$K_{\mathrm{e}_{-} \text {inside }}=\operatorname{IFFT}\left\{\left[\operatorname{FFT}\left(K_{\mathrm{e}_{-} \text {outside }}\right)^{-1}+G_{\mathrm{T}}\right]^{-1}\right\}$ 
This corrected electrode kernel $K_{\mathrm{e} \_ \text {inside }}$ can be further used to compute the electrode voltage by convolving it with the injected current $I_{\mathrm{e}}$ :

$V_{\mathrm{e}}=\left(K_{\mathrm{e}_{-} \text {inside }} * I_{\mathrm{e}}\right)$

However, since no electrode capacitance compensation was used in our experiments, the measured electrode + neuronal voltage $V_{\mathrm{e}+\mathrm{n}}$ and the computed electrode voltage $V$ were low-pass filtered by the electrode, but could be corrected using voltage deconvolution (Richardson and Silberberg, 2008) with:

$V^{\prime}=\tau \frac{\mathrm{d} V}{\mathrm{~d} t}+V$

Here by $\tau=R C$, with $R$ being the impedance at the lowest frequency and $C$ estimated from imaginary $\left(Y_{\mathrm{e}}\right)$ during the PNEC procedure.

After application of this voltage deconvolution to $V_{\mathrm{e}+\mathrm{n}}$ and $V_{\mathrm{e}}$, the neuronal voltage response could be computed as $V_{\mathrm{n}}=$ $V_{\mathrm{e}+\mathrm{n}}-V_{\mathrm{e}}$. The results of this procedure were applied offline to the responses of a phasic (Figure $5 \mathrm{C1}$, green traces) and a tonic $2^{\circ} \mathrm{VN}$ (Figure 5C2, green traces), measured during multi-sine stimulation (Figures 5C1,C2 black traces) with current amplitudes in the linear range, showed that the high-frequency electrode voltage had been removed leaving a low-frequency neuronal voltage, since the injected current was filtered by the neuronal time constant. Compensation of electrode voltage under noisy current stimulation is a demanding task for each electrode compensation mechanism: using a simple constant resistance $R$ for the estimation of the electrode voltage and thus subtracting $V_{\mathrm{e}}=R I_{\mathrm{e}}$, equivalent to standard bridge compensation, leads to insufficient electrode compensation (Figures 5C1,C2 red traces) since small errors in the estimation of the electrode voltage result in high-frequency electrode artifacts (compare with Brette et al., 2008, supplemental data). In addition, using the uncorrected electrode kernels $K_{\text {e_outside }}$ for offline compensation (Figures 5C1,C2 blue traces) causes an incorrect estimation of $V_{\mathrm{n}}$ since the overall electrode resistance is overestimated in this case.

In essence, this indicates that the PNEC procedure can be used for a direct estimation of the electrode kernel and offline subtraction of the electrode voltage in the time-domain. For some applications, such as voltage-, or dynamic-clamp experiments, an offline compensation, however, is not sufficient. Nevertheless it is clear that the PNEC procedure to determine the electrode kernel can also be used in combination with online AEC (Brette et al., 2008), as long as the electrode is measured twice: before entering a neuron and inside a neuron. It should also be noted, that for online AEC using the PNEC electrode kernel estimation it is more convenient to use capacitance compensation from the amplifier, as also suggested for normal AEC (Brette et al., 2008), which supersedes the voltage deconvolution step.

\section{DISCUSSION}

The new Piece-wise Non-linear Electrode Compensation (PNEC) method provides a way to take into account the non-linear effects of arbitrarily complex electrode properties in the frequency-domain, without online control or compensatory manipulations during the recording. This not only avoids the uncertainties involved in separating the effects of the electrode compared to those of the neuron, but also the frequent errors caused by improper online compensation, which is difficult if not almost impossible to correct afterwards.

In contrast to the Active Electrode Compensation (AEC) method described recently (Brette et al., 2008) the electrode properties in our PNEC method are not estimated from an electrode + neuron measurement, but are measured separately for each injected current step and corrected afterwards from the frequency-domain data, thus allowing the compensation of slow non-linearities caused by each current step. This compensation procedure is thus not only independent of electrode- and membrane-time constant ratios but also insensitive to resistance non-linearities of the electrode, which is not the case with AEC. Furthermore, using a simulation, it appears that the AEC electrode kernel estimation procedure is inaccurate especially when the electrodes show an inductive/resonating behavior, which however is not a problem for our PNEC procedure.

Frequency responses of neurons have been determined with low-resistant patch pipettes as well as with high-resistant sharp electrodes, but always using standard electrode compensation techniques. While this is not a problem for low-resistant patch pipettes, caution is advised when using high-resistant sharp electrodes and control experiments with the electrodes should be conducted prior to each experiment (see Moore et al., 1993). A previous work using high-resistant sharp electrodes (Gutfreund et al., 1995) states that their electrode impedance was frequency independent in the range of $0-50 \mathrm{~Hz}$, which suggests the following: first, the maximum frequency was sufficiently low in order not to cause errors due to distributed electrode RCs and second, since slices were used, the electrode tips were not immersed as deeply into the bath or brain tissue compared to whole brain recordings which reduces the low frequency inductive/resonating behavior (compare Figure 2A1, blue and green lines).

It is important to emphasize that the piece-wise linear electrode properties shown here, such as the resonant electrode behavior, should not be understood as a general description of all glass micropipettes but should rather be considered as an example showing that PNEC is capable of compensating any arbitrarily complex electrodes in the frequency-domain response.

It should also be noted that the offline PNEC method presented here was specifically developed for a frequency-domain analysis. Nevertheless, we could show that the PNEC framework can be used for determination of the electrode kernel and offline subtraction of the electrode voltage in the time-domain. Furthermore this kernel estimation procedure can be used with online AEC (Brette et al., 2008) as long the electrode is measured before entering a cell. The advantage of the combination of the two methods helps avoiding errors that result from the standard AEC electrode kernel estimation.

PNEC is a piece-wise linear method that requires an amplitude of the injected multi-sine current that does not trigger action potentials. Thus, the holding currents $I_{\text {hold }}$ have to be selected to drive the neuron either to a sub-threshold membrane potential or to a membrane potential where spiking does not occur due to sodium channel inactivation. As in other compensation methods, the present technique relies on combining distributed capacities of the electrodes. Based on the simulations, a frequency of $\sim 300 \mathrm{~Hz}$ 
is the upper limit for reliable neuronal impedance measurements with sharp, high-resistant electrodes using PNEC, which covers the important frequency range for most neuronal transfer functions. PNEC is capable of estimating neuronal transfer functions with high-resistant sharp electrodes and is thus a reasonable tool to correlate intrinsic membrane properties with synaptic signal processing in functionally intact whole brain preparations. The resulting neuronal representation in the frequency-domain can be used to analyze membrane properties and the different contributions of ion channels (Hutcheon and Yarom, 2000). It even is feasible to use these neuronal frequency responses to obtain Hodgkin-Huxley type models (Moore et al., 1995; Murphey et al., 1995; Booth et al.,

\section{REFERENCES}

Babalian, A., Vibert, N., Assie, G., Serafin, M., Mühlethaler, M., and Vidal, P. P. (1997). Central vestibular networks in the guinea-pig: functional characterization in the isolated whole brain in vitro. Neuroscience 81 , 405-426.

Beraneck, M., Pfanzelt, S., Vassias, I., Rohregger, M., Vibert, N., Vidal, P. P., Moore, L. E., and Straka, H. (2007). Differential intrinsic response dynamics determine synaptic signal processing in frog vestibular neurons. J. Neurosci. 27, 4283-4296.

Booth, V., Rinzel, J., and Kiehn, O. (1997). Compartmental model of vertebrate motoneurons for $\mathrm{Ca} 2+-$ dependent spiking and plateau potentials under pharmacological treatment. J. Neurophysiol. 78, 3371-3385.

Brette, R., Piwkowska, Z., Monier, C., Rudolph-Lilith, M., Fournier, J., Levy, M., Fregnac, Y., Bal, T., and Destexhe, A. (2008). High-resolution intracellular recordings using a realtime computational model of the electrode. Neuron 59, 379-391.

Erchova, I., Kreck, G., Heinemann, U., and Herz, A. V. (2004). Dynamics of rat entorhinal cortex layer II and III cells: characteristics of membrane potential resonance at rest predict oscillation properties near threshold. J. Physiol. 560, 89-110.

Gutfreund, Y., Yarom, Y., and Segev, I. (1995). Subthreshold oscillations and resonant frequency in guinea-pig cortical neurons: physiology and modelling. J. Physiol. 483, 621-640.

Hounsgaard, J., Kiehn, O., and Mintz, I. (1988). Response properties of motoneurones in a slice preparation of the turtle spinal cord. J. Physiol. 398, 575-589.
Hutcheon, B., and Yarom, Y. (2000). Resonance, oscillation and the intrinsic frequency preferences of neurons. Trends Neurosci. 23, 216-222.

Idoux, E., Eugene, D., Chambaz, A., Magnani, C., White, J. A., and Moore, L. E. (2008). Control of neuronal persistent activity by voltage-dependent dendritic properties. J. Neurophysiol. 100, 1278-1286.

Llinás, R., and Yarom, Y. (1981). Electrophysiology of mammalian inferior olivary neurones in vitro. Different types of voltage-dependent ionic conductances. J. Physiol. 315, 549-567.

Molecular Devices (2008). The Axon Guide, A Guide to Electrophysiology \& Biophysics Laboratory Techniques (Online). Available at: http://www. moleculardevices.com/pdfs/Axon Guide.pdf, Version 2500-0102 Rev. C.

Moore, L. E., Buchanan, J. T., and Murphey, C. R. (1995). Localization and interaction of N-methyl-Daspartate and non-N-methyl-Daspartate receptors of lamprey spinal neurons. Biophys. J. 68, 96-103.

Moore, L. E., Hill, R. H., and Grillner, S. (1993). Voltage-clamp frequency domain analysis of NMDA-activated neurons. J. Exp. Biol. 175, 59-87.

Murphey, C. R., Moore, L. E., and Buchanan, J. T. (1995). Quantitative analysis of electrotonic structure and membrane properties of NMDAactivated lamprey spinal neurons. Neural Comp. 7, 486-506.

Neher, E., and Sakmann, B. (1976). Singlechannel currents recorded from membrane of denervated frog muscle fibres. Nature 260, 799-802.

Pfanzelt, S., Rössert, C., Rohregger, M., Glasauer, S., Moore, L. E., and

1997; Tennigkeit et al., 1998; Saint Mleux and Moore, 2000; Roth and Häusser, 2001; Erchova et al., 2004; Taylor and Enoka, 2004; Idoux et al., 2008). In combination with synaptic activation (e.g. Pfanzelt et al., 2008) it is now possible to estimate the synaptic signal processing properties and transfer functions of individual neurons within an entire network.

\section{ACKNOWLEDGMENTS}

The authors acknowledge the help of Tobias Kohl in part of the neuronal recordings. This research was supported by the CNRS, CNES and BMBF (01GQ0440). Christian Rössert received a PhDgrant from the Bayerische Forschungsstiftung.

Straka, H. (2008). Differential dynamic processing of afferent signals in frog tonic and phasic secondorder vestibular neurons. J. Neurosci. 28, 10349-10362.

Richardson, M. J. E., and Silberberg, G (2008). Measurement and analysis of postsynaptic potentials using a novel voltage-deconvolution method. J. Neurophysiol. 99, 1020-1031.

Rössert, C., Glasauer, S., Moore, L. E. and Straka, H. (2008). White-noise analysis of central vestibular neurons with sharp electrodes in whole brain preparations. Soc. Neurosci. Abstr. 34 100.6

Roth, A., and Häusser, M. (2001). Compartmental models of rat cerebellar Purkinje cells based on simultaneous somatic and dendritic patch-clamp recordings. J. Physiol. 535, 445-472.

Saint Mleux, B., and Moore, L. E. (2000) Active dendritic membrane properties of Xenopus larval spinal neurons analyzed with a whole cell soma voltage clamp. J. Neurophysiol. 83, 1381-1393.

Straka, H., Beraneck, M., Rohregger, M., Moore, L. E., Vidal, P. P., and Vibert, N. (2004). Second-order vestibular neurons form separate populations with different membrane and discharge properties. J. Neurophysiol. 92, 845-861.

Straka, H., Biesdorf, S., and Dieringer, N. (1997). Canal-specific excitation and inhibition of frog second order vestibular neurons. J. Neurophysiol. 78, 1363-1372.

Straka, H., and Dieringer, N (1993). Electrophysiological and pharmacological characterization of vestibular inputs to identified frog abducens motoneurons and internuclear neurons in vitro. Eur. J. Neurosci. 5, 251-260.

Taylor, A. M., and Enoka, R. M. (2004). Quantification of the factors that influence discharge correlation in model motor neurons. J. Neurophysiol. 91, 796-814.

Tennigkeit, F., Schwarz, D. W., and Puil, E. (1998). GABA(B) receptor activation changes membrane and filter properties of auditory thalamic neurons. Hear. Res. 122, 18-24.

Wu, N., Hsiao, C. F., and Chandler, S. H. (2001). Membrane resonance and subthreshold membrane oscillations in mesencephalic V neurons: participants in burst generation. J. Neurosci. 21,3729-3739.

Conflict of Interest Statement: The authors declare that the research was conducted in the absence of any commercial or financial relationships that could be construed as a potential conflict of interest.

Received: 03 April 2009; paper pending published: 30 April 2009; accepted: 01 August 2009; published online: 20 August 2009.

Citation: Rössert C, Straka H, Glasauer S and Moore LE (2009) Frequency-domain analysis of intrinsicneuronal properties using high-resistant electrodes. Front. Neurosci. 3:64. doi: 10.3389/neuro.17.002.2009

This article was submitted to Frontiers in Neuroscience Methods, a specialty of Frontiers in Neuroscience.

Copyright ( () 2009 Rössert, Straka, Glasauer and Moore. This is an open-access article subject to an exclusive license agreement between the authors and the Frontiers Research Foundation, which permits unrestricted use, distribution, and reproduction in any medium, provided the original authors and source are credited. 